\title{
Where technology succeeds and fails in education
}

\author{
Kevin Wong ${ }^{1} \cdot$ Mahesh Thapa ${ }^{2}$ (D)
}

Received: 30 October 2020 / Revised: 25 February 2021 / Accepted: 9 May 2021 / Published online: 27 May 2021

(C) The Author(s), under exclusive licence to Springer-Verlag GmbH Germany, part of Springer Nature 2021

As the years go by we have seen an explosion in technological advances. Just in radiology, in the span of 40 years we have seen a transition from traditional film to digital radiographs and a marked increase in the use of CT and MRI. With this technological leap has come an interest in applying new technologies to education. PowerPoint has replaced traditional slide projectors and brought the ability to play cine clips. Then came websites such as Radiopaedia and STATdx, which gave learners encyclopedic volumes of knowledge at their fingertips. Recently we have seen audience response systems, social media and video-conferencing jump to the educational forefront.

We are in a veritable technological age and the question is not will or should we use new technology in education but rather how do we get the most out of the available technologies to benefit our learners. In the 1980s and 1990s, learners were separated into different types, such as auditory and visual, etc. This was meant to help educators tailor presentations to the specific learners' needs [1]. This, however, has been debunked in recent years with the realization that everyone learns through all styles [2]. The goal is to utilize these technologies in a way that benefits most learners.

In this article, we discuss and offer our opinions on several technologically innovative methods of education. We highlight both the positive and potential negative aspects of each.

\section{Group texts}

A group text is an excellent and effective method of immediate communication. One popular group-texting app is WhatsApp (WhatsApp, Mountain View, CA). At one of the

Mahesh Thapa

thapamd@uw.edu

1 Department of Radiology, Arkansas Children's Hospital, University of Arkansas for Medical Sciences, Little Rock, AR, USA

2 Department of Radiology, Seattle Children's Hospital, 4800 Sandpoint Way NE, MA.7.220, Seattle, WA 98105, USA authors' institutions, WhatsApp is used to provide unstructured education to trainees and for effective communication during call. For example, it is simple for a trainee to snap an image of a pediatric elbow radiograph, share it with her attending via WhatsApp, and ask whether the lucency she sees is a fracture or a growth plate. As long as there is no patientidentifying information, WhatsApp can be used by health care professionals [3]. At Duke University, the Department of Cardiology has successfully deployed WhatsApp to supplement fellow education. Participants in the group-chat share an interesting case and the group then engages in discussions regarding diagnosis and management plans with a goal to share individual case-based teachable moments with the entire fellowship and stimulate interesting discussion throughout the workday [4]. The Duke authors offer some helpful suggestions, two of which we feel are equally applicable for radiology education: (1) The platform should not be used in a punitive fashion and everyone should feel comfortable enough to give their opinions; (2) Once a case is presented, some time period (10-15 $\mathrm{min}$ ) should be allowed to pass before the correct answer is given. This helps facilitate a richer discussion. In addition to these suggestions, we recommend, when possible, providing several examples of the case being discussed and in different modalities. There are some potential drawbacks, however, to using group texts for education. One must be cognizant of the environment and refrain from using it in front of patients or in didactic settings because it might appear to be rude and insensitive. When using a phone-based system such as WhatsApp, the educator must realize that learners will be straining their eyes looking at their phone screens trying to see the finding, so image selection is key. This means when doing educational activities, the discussion should be less about the finding and more about the differential diagnosis and other aspects of the case.

\section{Audience response system}

Several studies have shown that an audience response system is an effective and engaging tool in medical education [5-9]. 
Kung et al. [7] found that surveyed radiology residents believed they learned better from lectures that incorporated an audience response system, felt more comfortable answering questions via an audience response system, and were more likely to attend a lecture that incorporated an audience response system. The authors also discovered that lecturers using an audience response system subjectively perceived that the trainees were more engaged in the lectures and that it was easier to judge the level of the audience's understanding and to prepare effectively for future lectures [7]. Utilizing an audience response system is not without its drawbacks, however. Lecturers have claimed that inserting questions can disrupt the flow of a lecture, that an audience response system requires more time investment and expertise, and that many systems only allow for multiple-choice or true/false questions $[8,10]$. In addition, the long-term effect on learning is controversial $[5,6,11]$. Awan et al. [5] found that when residents were examined at baseline and 8 months following on a series of musculoskeletal radiology lectures, there was no statistical difference in the test scores between the group that received instruction using an audience response system and the group that did not. Conversely, Rubio et al. [11] found that radiology trainees who were instructed using an audience response system did demonstrate statistically significant improvement in long-term (3 months) retention vs. the control group. Of note, a limitation not specific to audience response systems but to multiple-choice questions, in general, is that they confine the learner to pick the best or most correct choice, whereas in clinical medicine the answer is often a mix of answers or not presented as a list to choose from.

Several audience response systems are available, and our experience has been mostly positive. For radiology education, we found that the following systems offer the best combination of features and ease of use: Poll Everywhere (San Francisco, CA), Nearpod (Miami, FL) and Socrative (Edmonton, Alberta, Canada). We encourage the interested reader to explore each software and choose one that best fits his/her individual needs. We have the most experience with Poll Everywhere. For higher education instructors, the free version is robust, allowing for up to 40 audience members, which is plenty for most trainee lecture sessions. In addition, no sign-up is required of the students, who can participate via an app, website or text. Finally, Poll Everywhere offers convenient plugins for PowerPoint (Microsoft, Redmond, WA), Keynote (Apple, Cupertino, CA) and Slack (Salesforce, San Francisco, CA), helping to make adding this software a more efficient process for the lecturer.

\section{PowerPoint}

PowerPoint has supplanted the traditional projector slide show as a mainstay in education. There has been much debate about proper PowerPoint etiquette in the literature, and most universities even offer classes on how to make better PowerPoints. For example, authors have suggestions on the maximum number of words on a slide or lines of text per slide. The novice (and a few veteran) presenters often inundate the audience with text-filled slides, forcing the learner to read slides with tiny fonts, all while the presenter is talking about the slide [12-14]. This leads to people ignoring the presenter and just reading the slide, rendering the technology no better than reading a chapter in a book. On the flip side, when presenters themselves read the slide to the audience, they diminish engagement. What people fail to realize about this technology is just how much it can do beyond traditional slides. On a basic level, an educator can use cine clips, transitions and backgrounds that traditional projector lectures were unable to do. A pitfall of cine clips is when they play too fast or too slow. But within the software, the presenter can adjust the frame rate to make it play at the optimal speed. PowerPoint has done a very good job of integrating with other technologies such as audience response systems to allow for increased engagement throughout lectures. Additionally, hyperlinks can be embedded within the presentation, which allows the presenter to seamlessly skip to other parts of the talk, including the summary slides. This was most eloquently used in one of the authors' favorite lectures by way of a Jeopardy game. This was an interactive way to compete against other learners and learn the material needed.

\section{Social media (Twitter, Facebook and Instagram)}

Social media comprise numerous platforms, each with a slightly different audience. The big three are Twitter (San Francisco, CA), Facebook (Menlo Park, CA) and Instagram (Menlo Park, CA). Educators, institutions and companies are attempting to leverage the exponential growth of social media in innovative and novel ways [2]. Each platform has its own pros and cons in this educational venue. Social media can allow the user or institution to reach a much broader audience than they could otherwise through traditional lectures and national conferences. It allows us to connect with people from around the world on a daily basis and help people in underserved countries. The pitfalls, though, are many, and one must tread lightly when delving into such a public forum. First are Health Insurance Portability and Accountability Act (HIPAA) concerns, which must be addressed; images and text must be anonymized to ensure compliance. Using a vague history such as saying "infant" instead of giving an age and leaving out male or female unless relevant to the case are ways to stay safe. It is also imperative to omit extremely rare or very recent cases because the patient identity might be figured out based on disease and institution. 
Each platform lends itself to different learning opportunities. "Tweet chats" on Twitter have been used for journal clubs, allowing for people from all over the globe to participate [15]. Instagram has a number of accounts that give a caseof-the-day such as @PCHradiology and @thexraydoctor. It also has accounts dedicated to radiologic anatomy such as @ theradiologistpage. Facebook is a hybrid of Twitter and Instagram. Twitter is predicated on real-time tweets and responses, whereas Instagram is on the complete opposite end of the spectrum, allowing users to post and forget about the post. Facebook lives somewhere in the middle, allowing for more text than Instagram but less of a live component than Twitter. It has been used for educational blogs and to direct people to online educational resources through links. A recent addition to Facebook is the Facebook Live component, which allows users to have a livestream lecture on any topic that anyone on Facebook can view. Additionally, this video is saved to the person's page for viewing at a later time [16].

\section{Websites (Radiopaedia, STATdx)}

There has been an explosion in not only technology but also medical knowledge, with the projected doubling time being a mere 73 days [4]. Concurrently, innumerable websites are organizing and storing such easily accessible information. Smartphones and tablets are perpetually connected to the Internet, and it takes just seconds to search any information you want. Two of the largest websites for radiology are Radiopaedia [17] and STATdx [18]. These two websites have starkly different approaches for the same goal: to create a radiology encyclopedia. Radiopaedia is an open-access site that relies on volunteers to share their expertise and write entries about different disease processes. These entries are then edited and vetted by a dedicated group of mainly volunteers. Radiopaedia's entries tend to be short and to the point. These authors have found Radiopaedia to be a highly accurate and a very good source of digestible information. STATdx, on the other hand, is a subscription service that invites known experts to write entries on their areas of expertise. These entries tend to be very in-depth and, in one author's opinion, sometimes difficult to digest. Neither site is truly peerreviewed; however, the general consensus is that STATdx is more accurate than Radiopaedia. In these authors' experience, both sites are functioning at similarly high levels of accuracy.

These websites are not without shortcomings. Often articles are written by a single author, and rarely you find that author's personal opinions, particularly in fields/areas without consensus. Experts in the same field with different backgrounds might disagree about a specific disease process and this can be confusing to learners. Ensuring a website contains accurate material is paramount to a learner's success. We recommend evaluating the references listed at the end of each article to help judge how good the content on the website actually is. Additionally, many textbooks have online versions that learners can access through their university libraries. In our experience, most learners prefer reading websites over large textbooks because of their outline-style organization and multitude of examples.

\section{Video-conferencing (Zoom, Microsoft Teams, Cisco WebEx)}

With the coronavirus disease (COVID-19) pandemic going on, educators have been scrambling to figure out how to teach their learners while socially distancing. In comes the video conference. At their core, these software platforms are very simple: your group logs in and everyone can see everyone else on their screen and then someone can share his/her computer screen with the other members of the group to show them a PowerPoint or other educational content. There are three predominant platforms: Zoom (Zoom Video Communications, San Jose, CA), Microsoft Teams (Microsoft) and Cisco WebEx (Cisco Systems, San Jose, CA). Each of these platforms is slightly different and has different strengths and weaknesses. Zoom is the most user-friendly and allows you to see many people on the same screen, i.e. more than four. However, Zoom has also been plagued with some security issues and in its free form is not considered HIPAA-compliant. Microsoft Teams and Cisco WebEx are both considered HIPAA-compliant; however, they are not quite as userfriendly or as aesthetically pleasing as Zoom in one author's opinion. They also only allow for four people to be seen on the screen at the same time.

The difficulties one runs into with video-conferencing are the learners' understanding of the technology and proficiency using it (muting and unmuting themselves) and also the loss of in-person interaction. A good lecturer does not just stand behind a lectern and orate material; he/she walks around and interacts with the audience, reading the audience's cues to know whether to speed up, slow down or make other adjustments. When video-conferencing, these tasks are difficult to perform, and people tend to interact less as an audience and as a lecturer.

Some strategies to improve the interaction are, one, to have everyone turn on their webcams, including the presenter, which "humanizes" the presenter and the audience. Having good audio fidelity with any video presentation is also a must. It is relatively inexpensive to purchase a headset with a microphone. If your audience members do not have to struggle to hear the points you are making, they are more likely to stay tuned in. Also, utilizing functions built into the software such as the ability to draw on the slides can increase audience engagement. Using an audience response system with videoconferencing is another way to increase audience engagement 
throughout the lecture. Making use of the built-in chat function is also a great way to solicit questions without disrupting the lecture, allowing the lecturer to answer them at an appropriate time. These software platforms also have the ability for breakout sessions, which allows the lecturer to split a big group into smaller groups for discussion and then bring them back into the big group to finish the lecture. Another benefit of this technology is that you are able to record these sessions and learners are able to watch them as many times as they need to, and even at variable speeds, so as to ingrain the lesson into their memories.

Throughout this pandemic a number of institutions have banded together to put on visiting professor lecture series, which gives learners the opportunity to learn from worldrenowned experts from the comfort of their home. Without Zoom and similar platforms, teaching, clinical conferences and even national and international conferences would not have been possible. They have matured and come into their own because of this pandemic and are expected to have a lasting effect on the way the world functions going forward. One such impact in the realm of radiology has been on resident sign-outs. These platforms, with their screen-sharing abilities and the ability to give users control of the screen, have changed the day-to-day traditional readout sessions performed between staff and trainees. In our opinion, they are almost as good as in-person sign-outs at this point. They allow trainees to view their own screen - no more crowding around the attending's screen to try and catch a glimpse of what is being pointed out. Also, with the ability to allow the trainee to scroll through images, it is easier for the trainee to point out findings and for the attending to teach about those findings.

\section{Beyond residents and fellows: a note on medical students}

While we have mainly focused on how technology succeeds and fails in the education of radiology residents and fellows, we think it is also important to consider how we can leverage technology to continue educating our medical students. This demographic is crucial because these are the future radiologists and clinicians who will be utilizing radiology in their daily practice. Traditionally, they have observed behind attendings, residents or fellows and played a predominantly inactive role in education. COVID-19 has forced us to reimagine how we train our residents and fellows, and as such we should also consider how we can train our medical students. Of note, because these medical students grew up in this technological age, they tend to be very savvy when it comes to utilizing technology to its fullest. In our practice, we have integrated our medical students with our residents, meaning they attend daily resident lectures and virtual read-outs. Another great way to include medical students and improve their experience is to create online modules similar to the Cleveland Clinic pediatric radiology modules geared specifically for their level. For example, the Michigan State University College of Osteopathic Medicine in Lansing, MI, has done an incredible job creating interactive modules for medical students that involve looking at imaging and then annotating images and explanations.

\section{Conclusion}

Technology is both amazing and complicated. While it is supposed to make life and learning easier, in many instances it does not. However, the pandemic has thrust many technologies to the forefront of teaching and learning at a distance, and radiology is no different. Because most technologies have strengths and weaknesses in specific situations, we described a few of them here to help the pediatric radiology instructors using these technologies to choose the right technology or mix of technologies for the right situation and to use those technologies to their fullest potential.

Acknowledgments Special acknowledgment goes to Paul S. Lewis for his insightful thoughts on how to use technology to educate radiology residents.

\section{Declarations}

Conflicts of interest None

\section{References}

1. Fleming ND, Mills C (1992) Not another inventory, rather a catalyst for reflection. Improve Acad 11:137-155

2. Hillman T, Sherbino J (2015) Social media in medical education: a new pedagogical paradigm? Postgrad Med J 91:544-545

3. (2017) Is WhatsApp HIPAA compliant? HIPAA Journal. https:// www.hipaajournal.com/whatsapp-hipaa-compliant/. Accessed 27 Jun 2020

4. Kochar A, Rymer J, Samad Z et al (2018) Disrupting fellow education through group texting. J Am Coll Cardiol 72:3366-3369

5. Awan O, Dako F, Akhter T et al (2018) Utilizing audience response to foster evidence-based learning in a pilot study: does it really work? Cureus 10:e3799

6. Fergusson SJ, Aka JJ, Hennessy CM et al (2018) Examining the impact of audience response systems on student performance in anatomy education: a randomised controlled trial. Scott Med J 63: $16-21$

7. Kung JW, Slanetz PJ, Chen P-H et al (2012) Resident and attending physician attitudes regarding an audience response system. J Am Coll Radiol 9:828-831

8. Nicholson BT, Bassignani MJ (2009) Radiologist/educator knowledge of the audience response system and limitations to its use. Acad Radiol 16:1555-1560 
9. Awan OA, Shaikh F, Kalbfleisch B et al (2017) RSNA diagnosis live: a novel web-based audience response tool to promote evidence-based learning. Radiographics 37:1111-1118

10. Richardson ML (2014) Audience response techniques for 21 st century radiology education. Acad Radiol 21:834-841

11. Rubio EI, Bassignani MJ, White MA, Brant WE (2008) Effect of an audience response system on resident learning and retention of lecture material. AJR Am J Roentgenol 190:W319-W322

12. Penciner R (2013) Does PowerPoint enhance learning? CJEM 15: 109-112

13. Alpert JS (2019) So, you have to give a lecture - are you anxious? Am J Med 132:545-546

14. Collins J (2004) Education techniques for lifelong learning. Radiographics 24:1483-1489
15. Bolderston A, Watson J, Woznitza N et al (2018) Twitter journal clubs and continuing professional development: an analysis of a \#MedRadJClub tweet chat. Radiography 24:3-8

16. Kauffman L, Fishman EK, Mahesh M, Weisberg EM (2020) Breaking news: using Facebook live to transmit radiologic information quickly on a global scale. J Am Coll Radiol 17:899-902

17. Radiopaedia (2021) Radiopaedia website. https://radiopaedia.org/? lang=us. Accessed 11 Mar 2021

18. STATdx (2021) STATdx website. Elsevier. https://www.statdx. com/. Accessed 11 Mar 2021

Publisher's note Springer Nature remains neutral with regard to jurisdictional claims in published maps and institutional affiliations. 